\title{
Indígenas aislados. Aproximación a la incógnita humana de la Amazonía ecuatoriana
}

Fernando Paniagua Blanc*

\begin{abstract}
Resumen
Este artículo explora diversos aspectos de los grupos indígenas aislados de la AMAZONÍA ecuatoriana y, de MaNera especial, de aquellos QUe, de una forma genérica, SE han denominado Taromenani. SE ahonda EN LAS CARACTERÍSTICAS QUE CONFIGURAN SU ETNICIDAD: IDENTIDAD, LENGUA, TERRITORIO, RASGOS FÍSICOS Y antepasados. Pero además se plantean cuestionamientos Sobre su denominación, SObre la posibilidad de QUE SE TRATE DE UNA PLURALIDAd DE GRUPOS Y SOBRE SU VÍNCULO ETNOHISTÓRICO CON EL PUEBLO WAORANI.
\end{abstract}

Palabras claves: Taromenani - Tagaeiri - Waorani - aislados - Amazonía.

\section{Abstract}

THIS ARTICLE EXPLORES A VARIETY OF ASPECTS OF ISOLATED INDIGENOUS GROUPS IN THE AMAZON ECUADORIAN REGION, WITH SPECIAL ATTENTION GIVEN TO THOSE GROUPS GENERICALLY REFERRED TO AS TEROMENANI. WE WILL DISCUSS MORE DEEPLY THE CHARACTERISTICS WHICH DEFINE THEIR ETHNICITY: IDENTITY, LANGUAGE, TERRITORY, PHYSICAL FEATURES AND ANCESTORS. IN ADDITION, WE WILL POSE QUESTIONS ABOUT HOW THEY ARE DEFINED BY SOCIETY AT LARGE, THE ALTERNATE POSSIBILITY THAT INSTEAD THEY ARE PART OF A PLURALITY OF GROUPS AND THEIR HISTORIC LINK WITH THE WAORANI.

Keywords: Taromenani - TAgaeiri - Waorani - isolated - Amazonía.

* Doctor en el programa Historia y Arqueología, especialización en Antropología Americana, por la Universidad Complutense de Madrid. Correo electrónico: fernandopaniaguablanc@gmail.com 


\section{Introducción}

bundantes hipótesis y escasas aserciones son el bagaje inevitable de cualquier aproximación documental al conocimiento de los que, comúnmente, se han

1 denominado como pueblos no contactados, a los que instituciones nacionales e internacionales, investigadores de los mismos y responsables públicos insisten en la conveniencia de llamar Pueblos Indígenas en Aislamiento Voluntario (PIAV) (CIDH, 2013: 4) o simplemente Pueblos Indígenas en Aislamiento o Aislados (PIA) (Proaño y Colleoni, 2010: 7-8; Huertas, 2002: 21).

Un hecho que ha caracterizado el estudio de estos pueblos en el Ecuador en los últimos cinco años ha sido el componente ideológico. En un intenso debate público sobre el avance extractivista, estos grupos han pasado a ser protagonistas de una batalla de la que ellos -es seguro- no saben nada. Entre las muchas informaciones que los refieren, la mayoría han abordado el tema desde una perspectiva jurídica o política. Sin embargo, en este marco, hace falta estudios desde otros campos como la antropología o la historia, donde todavía las carencias son notables. Entre las pocas excepciones, no las únicas, quiero citar a Miguel Ángel Cabodevilla y a José Proaño, de quienes este artículo es sin duda deudor y espera ser la primera piedra de una continuación.

Es precisamente el desconocimiento cuasi absoluto sobre estos pueblos en muchos sentidos el que me ha impelido a iniciar el estudio de los mismos. Probablemente decir que, por encima de todo, se trata de una simple cuestión de curiosidad no sea correcto en un texto científico. Este sólo sé que no sabemos nada lo pone de manifiesto Cabodevilla, al subrayar que "no se sabe qué gente hay, cuánta hay, donde está ni quiénes son. No se ha hecho nunca un intento serio de conocimiento" (Vera, 2013).

El objetivo principal del presente trabajo es hacer un análisis de las características que definen la etnicidad de estos grupos. He seguido para ello la clasificación propuesta por Llorens (2002) y lo he aplicado desde una perspectiva amplia del término.

En lo que refiere a la identificación de los grupos, con el sin fin de problemas que esto representa en los grupos aislados, me serviré de un cuestionamiento terminológico para intentar hacer una aproximación a su identificación. El resto de características étnicas, a excepción de la ocupación del territorio, las analizaré desde una perspectiva comparativa con el pueblo Waorani.

Además, la primera parte del artículo, a fin de contextualizar el resto del texto, la dedicaré a hacer un muy somero recorrido de la historia de los Tagaeiri, para después mostrar la aparición del resto de grupos aislados a ojos de Waorani, investigadores y opinión pública.

Este artículo es el resultado de varios meses de búsqueda y análisis de información documental y de un amplio número de entrevistas realizadas a investigadores que han abordado el tema. Con posterioridad, durante el proceso de corrección, me he permitido también añadir algunas de las informaciones obtenidas a través de mi trabajo de campo, principalmente en comunidades Waorani.

\section{Los Tagaeiri y la aparición de otros grupos en aislamiento}

En 1993, Babe Imairi, jefe del clan waorani de Tigüino, secuestró a una joven durante una de las por lo menos cinco incursiones que realizó durante aquel año al que suponía territorio Tagaeiri. Las revelaciones hechas por Omatuki fueron recogidas por Miguel Ángel Cabodevilla, a quien los Waorani habían pedido acudir para mostrarle su hallazgo. Poco podía esperar el investigador que lo señalado por la joven removería el escaso conocimiento que aún para entonces se tenía sobre los pueblos en aislamiento. Omatuki, según recuerda Cabodevilla, explicó que había nacido en un grupo fruto de la mezcla de algunos Tagaeiri y un grupo diferente, al que comúnmente se ha denominado Taromenani. 


\begin{abstract}
"Durante algún tiempo, estos grupos habían intentado un intercambio de mujeres pacífico. Luego algo falló y un grupo de más al sur, liquidó a casi todos los hombres del viejo clan tagaeiri y se quedó con las mujeres". (M.A. Cabodevilla, comunicación personal, 18 de mayo de 2016).
\end{abstract}

De forma general se identifican dos grupos o pueblos de indígenas aislados en la Amazonía ecuatoriana: los Tagairi o Tagaeiri y los Taromenani o Taromenane (las denominaciones varían según los autores). Sobre los primeros existen innumerables relatos entre los Waorani que dan idea de su historia. Según W. Enomenga (comunicación personal, 11 de abril de 2016), surgieron como una escisión del gran clan de los Piyemoiri-Niwairi. La separación tuvo lugar por conflictos intraclánicos originados tras la muerte del líder del grupo, Niwa, en 1965, cuando se inició una lucha sangrienta entre los hijos de este y su hermano Kimontare por la sucesión en el poder. Tras varios enfrentamientos y muertos, la guerra finalmente concluyó en 1968, cuando los hijos de Niwa mataron a su tío y forzaron a sus descendientes, liderados por Tagae, a huir para no correr la misma suerte.

Precisamente en estos años (finales de la década de los 60), cuando el Instituto Lingüístico de Verano (ILV) estaba consiguiendo contactar y trasladar a la mayor parte de los Waorani a su protectorado en Tigüeno (Trujillo, 1981), los Tagaeiri, un pequeño grupo conformado entre 12 y 15 personas (Cabodevilla, 2010: 97), iniciaron una travesía con dirección al sur, posiblemente juntándose con otros Waorani que se resistían a las persuasivas técnicas de los misioneros evangélicos. Finalmente, se establecieron en torno a los ríos Mencaro y Tigüino, en donde permanecieron reacios y agresivos ante cualquier tipo de contacto tanto con cohuori (palabra waorani que designa a los ajenos a su pueblo) como con el resto de grupos Waorani ya contactados. (Coneolli y Proaño, 2012: 170-176; Cabodevilla, 1998: 281-283).

El ataque contra un grupo de exploración sísmica de la compañía CGG en 1977, que se saldó con la muerte de tres trabajadores, fue uno de los primeros de los muchos conocidos que el grupo de Tagae llevó a cabo después de separarse, principalmente contra trabajadores petroleros y madereros ilegales (Viteri, 2008: 173-197). Sobre los Tagaeiri se conocen datos ciertos sobre hechos concretos que han marcado sin lugar a dudas su devenir histórico, como la muerte de su líder Tagae durante el asalto a un grupo de petroleros en el río Shiripuno en 1984 (Tagliani, 2004: 106-107; Amunarriz en Cabodevilla, 1998: 30-31), confirmada con posterioridad por algunos waorani (Ibíd., 1998: 200).

Desde una perspectiva histórica, la principal duda que queda sobre este grupo, al que popularmente se conocía como el de los patas coloradas por la costumbre de sus miembros de pintar sus pies con achiote (Bixa Orellana), es la de determinar con exactitud qué ha ocurrido con él después de entrar en contacto con otro grupo en aislamiento. A tenor de lo que supuestamente señaló Omatuki, después de un proceso de fusión, miembros del otro grupo mataron a los hombres Tagairi, quedándose con las mujeres, por lo que se podría hablar de una absorción del grupo.

Esta tesis se vio en parte ratificada en el 2000 cuando Tihue, un waorani residente en Ganketapare, se encontró con un hombre que se identificó como Tagaeiri durante una de sus jornadas de cacería. El hecho sorprende por la lejanía de la zona con los territorios en los que se ha tenido constancia de la presencia de los Tagaeiri. Según la mujer de Tihue, el hombre había contado que su grupo había llegado hasta allí huyendo de otro grupo que les estaba atacando. Cabodevilla (2004b: 41-42) asegura que el nombre del tagaeiri referido es el de Huaihua (o Baiwa), el último de los grandes guerreros tagaeiri que, según Omatuki, quedaba vivo después que el resto muriesen durante el enfrentamiento con los hombres del grupo aislado al que se habían aliado o en accidentes en la selva (Ibíd., 1998: 295).

Aunque no dispongo de datos suficientes para determinar la posible desaparición o supervivencia de los Tagaeiri, sí parece claro un enfrentamiento de éstos con otro grupo en situación de aislamiento que se internó en su territorio. Un conflicto con este grupo pudo originar una huida y reubicación de los Tagaeiri en territorios más septentrionales, próximos a los ríos Tiputini y Tivacuno, en torno al año 2000. 
Después de que durante los primeros años de la década del 70 los misioneros del Instituto Lingüístico de Verano (ILV) hubiesen conseguido trasladar al $90 \%$ de la población waorani a su reducción en Tigüeno (Narváez, 1996: 36) y que, a partir de 1976, el Padre Alejandro Labaka hubiese logrado establecer relaciones pacíficas con el grupo waorani del río Dicaro, uno de los pocos que había conseguido mantenerse alejado de los evangélicos, se generalizó la idea de que los Tagaeiri eran el último y único grupo que quedaba en aislamiento en la Amazonía ecuatoriana (Santos, 1996: 199; Archivo del Vicaritao de Aguarico, 621). Las revelaciones de Omatuki supusieron un vuelco en este sentido. Lo señalado por la joven dejaba claro la existencia de, al menos, otro grupo aislado. Sin embargo, con anterioridad a su aparición, existían ya algunas pruebas que insinuaban esta posibilidad.

Entre los primeros en recogerla está el Padre Alejandro Labaka (2003: 122), quien en su diario de campo y coincidiendo con uno de las visitas que realizaba al grupo waorani del Dicaro, con fecha 21 de febrero de 1979, escribía:

"No he podido captar los nombres de los pueblos, pero me parece importante porque ha nombrado a los "cohuori", extraños al pueblo huaorani, y otros dos o tres más. Y entre éstos no ha nombrado ninguno de los otros grupos huaorani conocidos, a los cuales ha considerado dentro del nombre "huaorani". ¿Qué pueblos ha podido citarme, además de los huaorani y los cohuori...?"

Fue precisamente a raíz de la muerte del para entonces obispo, lanceado junto a la hermana laurita Inés Arango en 1987 mientras trataban de hacer contacto con un grupo Tagaeiri próximo al río Tigüino, cuando desde el vicariato de Aguarico se profundizó en el conocimiento sobre el pueblo waorani y los grupos en aislamiento. Misioneros como Juan Carlos Andueza, Juan Santos, Lino Tagliani o el ya citado Miguel Ángel Cabodevilla, entre otros, iniciaron a partir del trágico acontecimiento una investigación intensiva que, coincidiendo temporalmente con la incursión de estos grupos en los territorios Tagaeiri, permitió obtener las primeras pistas sobre ellos. Precisamente a esta etapa inicial de la investigación se refiere varios años después Cabodevilla (2004b: 36):

\footnotetext{
"Informaciones recogidas durante años con muy diversos Waorani que habitaban o cazaban por las cuencas de los ríos Yasuní, Nashiño, Cononaco y Shiripuno, entre otros, nos hablaban de huellas características, monos muertos por virotes extraños, en fin, señales varias de clanes no contactados".
}

Uno de los documentos más significativos y que mejor atestigua el hecho de que se empezaba a tener indicios de la existencia y ubicación de grupos en aislamiento diferentes a los Tagaeiri es un mapa dibujado por Tagliani durante un viaje exploratorio que realizó junto a Babe en 1992. En el dibujo se puede ver claramente como en la parte inferior, correspondiente a la zona situada al sur del río Mencaro, Tagliani escribe: “Taromenane?” (Ibíd., 2004: 107).

Cabodevilla asegura, sin embargo, que con anterioridad a la aparición de Omatuki, la existencia de grupos en aislamiento diferentes a los Tagaeiri era una simple "cábala" (comunicación personal, 19 de mayo de 2016). Merece la pena, en este sentido, hacer mención a un libreto explicativo publicado por el Ministerio del Medio Ambiente (1999) con motivo de la puesta en marcha de las zonas intangibles en el que, además de las declaraciones de Omatuki, se hace referencia a unos enfrentamientos en 1992 entre trabajadores dedicados a la exploración sísmica y un grupo de Waorani no contactados como la primera prueba de la existencia de grupos aislados diferentes a los Tagaeiri. Es llamativo observar que, entre el resto del corpus al que he podido tener acceso que hacen referencia a este tema investigativo, en ninguno se hace mención a este enfrentamiento en 1992. Se entenderá, por ende, la sospecha no sólo de que petroleros y gobierno habrían sabido sobre la existencia de estos grupos antes de 1993, sino que, además, han llevado a cabo investigaciones propias de las que, incomprensiblemente, poco o nada ha salido a la luz pública. 
Más allá del cómo y cuándo desde la sociedad mayor se empezó a conocer la existencia de este nuevo grupo (o grupos) en aislamiento, el hecho de que ésta fuera confirmada abrió una serie de interrogantes que han centrado la investigación hasta la actualidad, muchos aún sin resolver.

\section{Los últimos grupos encontrados: ¿Taromenani?}

Son escasos y poco fiables los datos a los que se puede acceder que ofrezcan una idea sobre la auto-identificación de estos grupos. He tenido la oportunidad de preguntar en repetidas ocasiones a Cabodevilla sobre la posibilidad de que Omatuki utilizase de forma literal la palabra "Taromenani" para referirse a su grupo de procedencia. La respuesta siempre ha sido la misma: "no lo recuerdo con exactitud" (comunicación personal, 23 de julio de 2015 y 18 de mayo de 2016).

El etnónimo Taromenani, con posterioridad a 1993 y hasta la actualidad, se ha generalizado para referirse a los grupos aislados que eran diferentes de los Tagaeiri. A falta de tener un conocimiento aproximado sobre la auto-denominación de los mismos, resulta en extremo difícil hacer una determinación sobre la validez de su utilización.

En la memoria colectiva Waorani se recogen varios nombres que refieren a grupos que vivían en la selva y con los que éstos mantenían conflictos antes de ser contactados. Entre los que se han registrado con más frecuencia están Iwene, Taromenani, Taromenga, Wiñatari, Menkatape, Oñamenane (W. Enomenga, comunicación personal; 16 de abril de 2016).

Ante esta variedad de nombres, Proaño (2010: 10) plantea dos opciones: que el término Taromenani se haya convertido entre los Waorani en un "genérico" para definir "una alteridad hacia otros grupos" y, por tanto, sea posible la existencia de grupos aislados diferenciados identitaria y étnicamente; o, por el contrario, que los diferentes grupos Waorani "definan con distintos nombres a un mismo grupo".

El hecho de que el nombre de Taromenani fuese uno de los más utilizados entre Waorani e investigadores en los años inmediatamente anteriores al secuestro de Omatuki (ver Cabodevilla, 2004: 93 y 107) para referirse a grupos con los que supuestamente aún no se había tenido ningún tipo de contacto y sobre los que su existencia ni siquiera estaba confirmada es aclaratorio por cuanto parece dar cabida a la hipótesis de que la denominación de Taromenani, que en el pasado habría identificado a un grupo bien definido, puede haber sido utilizado con posterioridad como un genérico para designar a una pluralidad de grupos étnicos que los Waorani identifican como ajenos. Sin embargo, tampoco cabe descartar la posibilidad de que, como parece entenderse por algunos testimonios waorani (E. Ima y Wemane), la denominación Taromenani haga referencia a uno de los principales grupos rivales con los que compartían territorios los Waorani en el pasado y que sean descendientes de este grupo los que incursionaron en las zonas de asentamiento Tagaeiri, primero, y se expandieron posteriormente.

Mis consultas a waorani sobre la existencia real de los grupos citados señalan la consideración general de que todos forman una unidad étnica en la actualidad. "Son lo mismo", ha sido la respuesta más frecuente. Sin embargo, varios informantes (W. Enomenga, W. Ahua) explicaron que el nombre de Wiñatari es con el que se identificaba en el pasado a los Taromenani.

El Ministerio de Medio Ambiente (1999: 9) aseguraba que, según algunos Waorani, además de Tagaeiri y Taromenani: "existen varios grupos más que no han sido contactados, y que viven bosque adentro. Sin embargo, no existen datos que puedan confirmar o descartar la presencia de estos otros grupos en la zona".

Mucho más tajante se muestra Smith (1996:120) al afirmar la existencia de dos grupos étnicamente diferenciables de los Taromenani: los Oñamenanes y Wiñatare. Pero este no tardó en encontrar la contra por parte de Lara y Rivas (2001: 72), quienes advierten que "ninguno de los Waorani consultados hasta el momento por nosotros, ni ninguna otra publicación confirman los datos mencionados por este autor [Smith]". 
Entre las informaciones que juzgo más precisas está la recogida por Cabodevilla, de Ompure, un anciano Waorani de la zona del río Yasuní que vivía apartado en la selva y mantenía relaciones esporádicas con algunos miembros de grupos en aislamiento:

"él [Ompure] distinguía claramente tres grupos diferentes. Había un grupo que, efectivamente, estaban mezclados con Waorani, fueran Tagaeiri o fueran otros; después había otros grupos, unos clanes de los Taromenani; y creía que quedaba otro grupo sin contacto de Waorani, no Taromenani, que estaban en la parte de la frontera con Perú". (M.A. Cabodevilla, comunicación personal, 23 de julio de 2015).

Ante la imposibilidad de llevar a cabo una identificación exógena directa y precisa sobre estos grupos, ha sido frecuente acudir a los Waorani. Cabodevilla (2004) hace referencia al pasaje de un texto de Tessman (1930) en el que se cuenta como el antropólogo encuentra a dos prisioneros en una hacienda del Napo que se autodenominaban "Huaos" y que, aunque habían olvidado casi todo su idioma, al ser preguntados por otros grupos cercanos, alcanzaron a citar dos: "Tarominca" y "Ereminca" (Ibíd.: 151). El descubrimiento, además de ser importante por tratarse del documento más antiguo de los que he podido tener noticia en el que se refiere a los Waorani, es revelador en el tema que nos ocupa por referir la denominación Tarominca, fácilmente semejable a la actual Taromenane. Se debe tener en cuenta que, aunque la obra de Tessmann fue publicada en 1930, el encuentro entre el antropólogo y los indígenas fue, sin duda, anterior; y los recuerdos de éstos aún más, lo que nos da idea de que, aproximadamente a principios del siglo XX, Waorani y Taromenani eran grupos vecinos y que se identificaban diferenciadamente.

Entre las formas genéricas de identificación que algunos Waorani han utilizado para referirse a estos grupos, varios autores han señalado el término Warani. Tagliani (2004: 45) explica que los Waorani utilizan para referirse a gente que habla la misma lengua pero "potencialmente enemiga porque no pertenecen al mismo clan". Proaño (2010: 19) aclara que significa "otro" en Waotetero; y Cabodevilla dice que al denominarlos así los Waorani pretenden decir que son "otros pero iguales" (comunicación personal, 23 de julio de 2015). Al referir a estos grupos aislados como Warani y no como Cohuori, forma en que los Waorani denominan a todos aquellos que no son parte de su pueblo, se puede intuir una vinculación étnica, sin embargo, en ningún caso se trata de una prueba concluyente.

\section{Los aislados de la Amazonía ecuatoriana: ¿Waorani?}

Pablo Ahua asegura que durante la estancia de Omatuki, entre los Waorani de Tigüino se fijaron en algunos rasgos que les llamaron la atención. Su forma de hablar les resultó extraña y con frecuencia tenían problemas para entender lo que decía; además, su piel era mucho más clara y tenía los ojos muy achinados (P. Agua, comunicación personal, 17 de abril de 2016). Cabodevilla (1998: 286) señala también las diferencias en el pelo, muy largo y abundante, y la forma de su corte, "con un amplio flequillo hasta la altura de las cejas, casi inexistentes; también se había depilado las sienes".

A las lingüísticas y fenotípicas, se añadieron después otras variaciones en la cultura material. El 22 de noviembre del 2000, durante el rescate de los cadáveres de dos indígenas Kichwas que habían sido lanceados en el río Curaray, algunos Waorani presentes indicaron que las lanzas eran diferentes a las suyas (Lucas, 2000).

Además de un ingente número de muertos sobre el que las cifras varían (Vera, 2007; Cabodevilla, 2010), la matanza ejecutada en mayo de 2003 contra miembros de un grupo en aislamiento por algunos waorani como venganza por la muerte de Omene diez años antes -y posiblemente también para favorecer negocios madereros en la zona (Coneolli y Proaño, 2012) dejó una gran cantidad de pruebas materiales. Éstas, que los atacantes extrajeron de la casa 
donde realizaron la matanza al sur del río Mencaro, muestran algunas diferencias importantes en la cultura material del grupo aislado y los Waorani. Andueza (Cabodevilla, 2004b: 79), tras encontrarse con los Waorani implicados en la matanza pocas horas después, comentaba: "Cuentan también las diferencias en el lenguaje, las terminaciones de la bodoquera, las estrías de la lanza, la diferente medida de la hamaca; sin embargo, se puede apreciar que es mucho más lo parecido que lo que les distingue".

Un cuestionamiento frecuente respecto a los últimos grupos conocidos en situación de aislamiento en la Amazonía ecuatoriana es el de su vinculación etnohistórica con el pueblo Waorani. Junto con la proximidad geográfica y el papel que los waorani han desarrollado como fuente de información, esta supuesta vinculación es la que ha justificado de alguna manera que los intentos por abordar una identificación y estudio etno-cultural de los grupos aislados en los últimos años se haya realizado, en la práctica totalidad de los casos, desde una perspectiva comparativa con el pueblo Waorani, atendiendo a sus similitudes y, sobre todo, a sus diferencias.

La informal y poco precisa pregunta ¿son Waorani? encuentra, entre investigadores y documentos, respuestas variadas. Sobre los Tagaeiri hemos señalado que se originaron como una escisión de otro grupo Waorani por lo que no caben dudas sobre su vínculo histórico. Es evidente que no presentan diferencias fenotípicas y que mantienen lazos de parentesco con el pueblo Waorani, Asimismo, entre las pruebas encontradas durante los años en que ocuparon el territorio entre los ríos Menkaro y Tigüino, no se observó que desarrollaran diferencias significativas en su cultura material con respecto a la de los Waorani con anterioridad al contacto. En este sentido, las principales diferenciaciones entre Tagaeiri y Waorani provendrían de consideraciones identitarias derivadas de su diferenciado devenir histórico después de que, mientras los Waorani se contactaron con la sociedad mayor, éstos permanecieron en aislamiento.

Más complejas son las consideraciones sobre esta vinculación etnohistórica con el resto de grupos en aislamiento. Coneolli y Proaño (2012: 170) afirman que: "Los denominados Taromenane son un grupo lingüística y culturalmente relacionado a los Waorani, manteniendo características en la cultura material (por ejemplo, las medidas y formas de las lanzas y bodoqueras) y lingüística (las terminaciones de las palabras)".

Rogalski (2003: 159, en: Cabodevilla, 2004) señala otros posibles vínculos de los grupos en aislamiento: "son emparentados con los Arabela y los Waorani. Sin embargo, existe también la posibilidad de que algunos de ellos sean descendientes de los Abijira, considerados pueblo extinto".

\section{Cultura material}

En una primera valoración de los objetos que el grupo de waorani que llevó a cabo la matanza en mayo de 2003, Cabodevilla asegura que:

"La hamaca era de diferente hechura; también el grosor, disposición de los garfios, labrado de las lanzas. Pero las innovaciones más convincentes residían en la cerbatana. Ese singular acoplamiento para la boca del soplador de dardos no podía haberse creado por casualidad, si tampoco era un invento casual o cercano. Tampoco la parte opuesta, en la salida de la flecha, más parecida a la de otros grupos [que al Waorani]. Eso sí, se trataba de la omena wao sin duda; del todo original y diferenciada a cualquier otra en la Amazonía entera. Sin embargo, esas variantes hablaban probablemente de un grupo afín, pero separado por largo tiempo, capaz de producir variaciones y de mantenerlas en aislamiento" (Ibid., 2004b: 37-38).

Sin embargo, la diferencia que más ha llamado la atención y que ha sido más estudiada está en las lanzas. Después de observar una de las lanzas obtenidas en la casa de uno de estos grupos en aislamiento tras el ataque de 2013 (Aguirre y Cabodevilla, 2013) y comparándola 
con lanzas waorani y con una de las Tagaeiri que se conserva de la muerte de Labaka en el museo de la Catedral del Coca, es claro que presentan diferencias notables. Si bien todas están fabricadas con madera de chonta (Bactris Gasipaes), la de estos grupos es más larga y considerablemente más gruesa. Además, la punta presenta una forma diferente, mucho más afinada.

A conclusiones parecidas llegan Coneolli y Proaño (2012: 186) después de analizar las lanzas utilizadas durante un ataque registrado en la comunidad de colonos de Los Reyes en el que fallecieron una mujer y dos de sus hijos: "La medida longitudinal de las lanzas y su ancho, así como los dientes, son más profundos y largos que las lanzas de los Waorani.

Cabodevilla señala también una diferencia entre los materiales con los que fabrican los canastos. Según el investigador, mientras que los grupos aislados los fabrican con bejucos, los Waorani antes de ser contactados "no sabían hacerlos de esa manera, sino sólo utilizando hojas" (M.A. Cabodevilla, comunicación personal, 23 de julio de 2015). En este sentido, sin embargo, Wane Enomenga (comunicación personal, 11 de abril de 2016) aseguró recordar que eran frecuentes las dos formas de fabricación entre los Waorani con anterioridad al contacto.

Sobre las casas, Villaverde (comunicación personal, 3 de septiembre de 2015) asegura que son exactamente iguales. Por el contrario, otra fuente (anónimo, comunicación personal, 25 de agosto de 2015) recalca que las de los grupos aislados son más grandes y, a diferencia de las waorani, presentan espacios diferenciados en su interior. Cabodevilla (comunicación personal, 23 de julio de 2015), apoyándose en fuentes waorani, recalca que las formas de construcción son diferentes.

Otro aspecto diferenciador en el marco de la cultura material es la utilización de expansores lobulares en las orejas, que eran comunes entre los Waorani, pero no lo son entre los aislados. Así, Katy Álvarez (comunicación personal, 15 de septiembre de 2015) asegura que los miembros de grupos aislados no los utilizan, mientras que Cabodevilla (comunicación personal, 23 de julio de 2015) advierte que algunos sí, pero son mucho menos comunes que entre los Waorani antes del contacto.

\section{Lengua}

La coincidencia lingüística ha sido abducida con frecuencia para defender una vinculación etnohistórica entre los grupos que actualmente permanecen aislados y los Waorani. En este sentido, se sabe que los aislados, al menos con los que se ha tenido algún tipo de contacto hasta el momento, hablan variantes del wao terero. Además de por los casos ya señalados de Ompure y Omatuki, son conocidos algunos otros ancianos waorani que habrían tenido algún tipo de comunicación oral con ellos. Asimismo, existen referencias de conversaciones entre aislados y Waorani que se encuentran en la selva durante jornadas de cacería. No obstante, con frecuencia los Waorani han señalado las dificultades para entenderlos, que se pueden derivar de variaciones dialectales.

"Su idioma es el mismo con variantes en la pronunciación, en las palabras y en algunos términos" (M.A. Cabodevilla, comunicación personal, 23 de julio de 2015). Esta coincidencia lingüística sería en extremo reveladora sobre la vinculación etnohistórica entre waorani y los grupos aislados si, como fue creencia común, se tratase de una lengua aislada. Esta idea surgió de las dificultades para clasificarla y encontrarle parentescos que encontraron los lingüistas del ILV, que no pudieron establecer ningún tipo de relación con ninguna otra de las familias lingüísticas presentes en Ecuador. En un informe realizado conjuntamente por el Ministerio de Educación y Cultura e ILV (1975 en: Narváez, 1996: 33) se referencia lo siguiente: "Para McQuown (1955) el huau está consignado como ' sin clasificación', aunque para Steward y Faron (1959: 22-23) y Swadesh (1959) puede agruparse según filos más comprensivos". 
Frente a esto, la clasificación del lingüista Joseph Greenberg (1987) incluye al wao terero en una unidad más a la que denomina Urarina-Waorani (Itucale-Sabela), dentro de la cual se encontrarían también la lengua Urarina, hablada por el pueblo del mismo nombre en la provincia peruana de Loreto, y el Omurano o Mayna, lengua del gran grupo Mayna, extinta alrededor de 1958 (Adelaar, 2004: 447). Aunque la clasificación realizada por Greenberg ha suscitado innumerables críticas y polémicas, parece que podría ser una buena fuente para un estudio de vínculos etnohistóricos tanto de los grupos aislados como de los mismos Waorani. En esta misma línea, Álvarez asegura que "la lengua del grupo Taromenane, es sólo una hipótesis, pero parece muy cercano al tronco lingüístico zapaorano" (Comunicación personal, 15 de septiembre de 2015).

\section{Rasgos físicos}

La observación de la fotografía conservada de Omatuki (Cabodevilla, 2004: 204) puede resultar aclaradora. En ella se puede observar cómo la joven es más grande y corpulenta de lo que usualmente son las mujeres Waorani, tiene la piel más clara y un corte de pelo diferente.

Como señalé con anterioridad, un aspecto que con frecuencia ha llamado la atención entre Waorani e investigadores son las supuestas diferencias físicas de los miembros de grupos aislados respecto a los primeros. Proaño (2010: 18), haciéndose eco de algunos testimonios de Waorani, lo expresa de la siguiente manera: "Son grandes, fuertes, astutos (...) son más fuertes y peligrosos que los Waorani". Además de su mayor tamaño, otros aspectos que los Waorani suelen resaltar al hablar de ellos, es su diferente tonalidad de piel, más clara, o la diferencia en la forma de los ojos, más rasgados.

Cabodevilla ha ratificado algunas de estas diferencias al asegurar que es cierto que "algunos de los que se han visto son robustos, son fuertes, más grandes, tienen más resistencia para la cacería", pero les resta importancia al señalar que lo que dicen los Waorani sobre los miembros de estos grupos es "exactamente lo mismo que decían los Naporunas sobre los Waorani hace unos años". Respecto al color de piel, Cabodevilla explica que "son más blancos porque no van al rio, porque no salen al sol, porque están siempre bajo la selva" (comunicación personal, 23 de julio de 2015).

Frente a esta posible exageración de las características físicas por parte de los Waorani, existen otros testimonios que las respaldan. Gilder Moreira, un maderero que sobrevivió al ataque de un grupo aislado en abril de 2006, dijo que "eran muchos hombres y mujeres, blancos y altos, no se parecían a los Waorani” (Vega, 2007). Lo mismo apuntó Ángel Velásquez, otro de los madereros implicados en el incidente: "eran unos blancos, altos, pelo largo y desnudos" (TVECUADOR, 2013).

\section{Antepasados comunes}

Los miembros del grupo de los Tagaeiri, como escisión del clan Waorani de los PiyemoiriNiwairi, comparten antepasados comunes y lazos de parentesco con miembros del pueblo Waorani. Sin embargo, entre los documentos revisados, las entrevistas a investigadores y el trabajo de campo realizado con Waorani, resultan muy escasas las referencias sobre la existencia de antepasados comunes entre éstos y los miembros pertenecientes a grupos aislados.

Merece la pena mencionar que algunos autores hablan de lazos de parentesco entre Waorani y Taromenani. Así, Trujillo (2001: 40-41) asegura que Wiñeycamo, mujer de Kay Aiwa, jefe de la comunidad Waorani de Kawymeno, "resultaría ser del grupo de Tarome". Cabodevilla (1998: 308), por su parte, señala que "el papá de Kemperi robó una mujer taromenairi y con ella tuvo varios hijos antes de que desapareciera; también Inihua (padre putativo de Alejandro Labaka) tiene familiares entre ellos". En cualquier caso, no he podido ratificar ninguna de estas afirmaciones y no parecen demasiado fiables. 


\section{Territorio histórico, migraciones y territorio actual}

Sobre el remoto origen geográfico tanto de Waorani como de los otros grupos en aislamiento, Villaverde (2012: 20), recoge el testimonio de Bai Ima, un anciano waorani: "Nuestro origen es del Perú, los papás y los abuelos contaban que vinieron de allí. Mi papá me dijo que, cuando vivíamos en Perú, [Los Taromenani] se iban llevando a la gente, secuestraban a la gente y se iban llevando más y más".

En un pasado más reciente, sabemos que los Tagaeiri, al escindirse en torno a 1967 y 1968 del clan Waorani Piyemoiri-Niwairi, situado en un territorio comprendido entre el río Tiputini y el Tivacuno, migraron hacia el sur hasta ubicarse en un territorio próximo a los ríos Menkaro y Tigüino y, por las zonas donde se registraron ataques y avistamientos en los años 70 (Viteri, 2008), es posible que llegasen a ocupar también la cuenca de los ríos Shiripuno y Cononaco Chico. Si damos por cierto los testimonios de Omatuki en 1993 y Tihue en 2000, quien se los encontró en las proximidades de la parte septentrional de la vía Maxus, después de haber entrado en conflicto con los otros grupos en aislamiento que se introdujeron en su territorio, los Tagaeiri habrían migrado de nuevo, regresando a zonas próximas a donde se originaron al separarse del clan Piyemoiri-Niwairi.

Sobre los otros grupos en aislamiento, Cabodevilla, (2004: 151), recordando la vecindad con los Taromica a la que se referían los dos Waorani encontrados por Tessmann (1930) en una hacienda del Napo, deduce que Waorani y Taromenani compartían territorio a principios del siglo XX en las cuencas bajas del Yasuní y el Tiputini, aunque también llegando hasta el Tivacuno.

Álvarez sostiene que, con la avanzada cauchera a principios del siglo XX, los Taromenane se habrían escondido "en las cabeceras de los ríos: en dirección hacia el río Tigres, hacia la frontera con el Perú" (comunicación personal, 15 de septiembre de 2015). Esta tesis la confirman Coneolli y Proaño (2012: 173) a través de un mapa en el que recalcan la existencia del grupo Taromenani en los territorios en torno a la frontera oriental entre Ecuador y Perú en 1958.

Refiriendo épocas posteriores, Wane Enomenga (comunicación personal, 14 de abril de 2016) relata como su padre, Name, durante el camino que el grupo de los Piyemoiri realizó desde su territorio tradicional en las proximidades del Tiputini hasta la reducción que el ILV tenía en Tigüeno, en los años 1968 y 1969, señaló dos lugares en los que había que tener precaución por la presencia de Taromenani. Uno de los lugares señalados se sitúa entre la margen derecha del río Tivacuno, próximo a los ríos Yasuní y Yamino; mientras que el otro estaría algo más al norte, en la margen izquierda del Tivacuno.

Pese a que parece probable que Waorani y Taromeani compartiesen territorios en el pasado, el hecho de que los segundos no fuesen identificados por el ILV durante su proceso de contacto evangelizador en la zona señalada parece apuntar a una temprana separación. Cabodevilla (comunicación personal, 19 de abril de 2016) plantea la posibilidad de que después de algún conflicto importante con los Waorani, los Taromenani se viesen forzados a alejarse de la zona, ocupando territorios más al este.

Sobre cómo con posterioridad estos grupos en aislamiento podrían haber penetrado en el territorio Tagaeiri existen dos hipótesis: Cabodevilla (2010: 120) señala que el contacto con grupos Tagaeiri se produjo entre 1990 y 1991, cuando miembros de estos grupos aislados, procedentes de zonas más orientales, avanzaron por el valle del río Nashiño y penetraron en los territorios Tagaeiri, contactándose con un grupo de éstos últimos en las proximidades del río Tigüino. Por el contrario, en atención a lo que apuntó Omatuki (Cabodevilla, comunicación personal, 23 de julio de 2015), estos grupos llegaron desde el sur. El mismo Cabodevilla (1998: 308) en otro momento, parece darle la razón al indicar que "los taromenani han sido siempre la amenaza al sur". En cualquier caso, después de haber accedido a estos territorios -y tal vez persiguiendo a los Tagaeiri- estos grupos también se expandieron hacia el norte. 
En los últimos años y en atención a su ubicación geográfica, algunos estudiosos e instituciones han optado por hacer una división de los grupos en aislamiento. La más interesante de estos grupos según su ubicación geográfica es la que hacen Coneolli y Proaño (2012: 172, 174 y 175), quienes apuntan la existencia de 3 grupos: uno al que denominan en el Cononaco Chico, otro al que denominan Cuchinyacu (aunque ocuparía un espacio muy amplio en torno al río Curaray hasta la frontera con Perú) y, finalmente un grupo en torno de los ríos Tivacuno y Yasuní.

En la actualidad, a través de datos del Ministerio de Justicia a los que he podido tener acceso en una presentación sobre pueblos indígenas en aislamiento organizada por la Fundación Alejandro Labaka en la ciudad del Coca (24 de mayo de 2016), se apunta a la existencia de dos zonas de ubicación bien diferenciadas. La primera ocupa un espacio vertical de aproximadamente 80 kilómetros entre la margen izquierda del río Curaray hasta la margen derecha del río Tivacuno; mientras que transversalmente se extiende aproximadamente entre la margen derecha del río Tiri hasta una línea imaginaria situada entre 20 y 25 kilómetro de distancia de la vía Auca. Pero además esta zona presenta en su parte meridional un brazo hacia el oeste de aproximadamente 30 kilómetros en las dos márgenes en torno al río Mencaro (Cunchiyaku).

La otra zona definida por el Ministerio de Justicia presenta una forma ovalada, con una leve inclinación noreste-suroeste. El eje mayor mide algo menos de 50 kilómetros y el menor ronda los 25. Se encuentra ubicada entre los ríos Curaray y Nashiño, situándose su centro aproximado a unos 25 kilómetros de la comunidad Waorani de Bameno.

\section{Bibliografía}

Cabodevilla, Miguel Ángel. 2008, Zona intangible: ipeligro de muerte!, CICAME, Quito.

2004, El exterminio de los pueblos ocultos, CICAME, Quito.

1998, En la región del olvido. Crónicas amazónicas 1984-97, CICAME, Quito.

Cabodevilla, Miguel Ángel y Aguirre, Milagros. 2013, Una tragedia ocultada, Fundación Alejandro Labaka, Quito.

Cabodevilla, Miguel et al. 2004b, Tiempos de guerra: Waorani contra Taromenane, Abya-Yala, Quito.

Castro, Germán. 1999, La noche de las lanzas: muerte de un obispo español en el Amazonas, Planeta, Barcelona.

Chávez, Gina. 2003, "Muerte en la Zona Tagaeiri Taromenane: justicia occidental o tradicional", en: ICONOS. Revista de ciencias sociales (17), Quito.

Comisión Interamericana de Derechos Humanos. 2013, Pueblos indígenas en aislamiento voluntario y contacto inicial. Recomendaciones para el pleno respeto a sus derechos humanos, IWGIA.

Greenberg, Joseph. 1987, Language in the Americas. Standford University Press.

Huertas, Beatriz. 2002, Los pueblos indígenas en aislamiento. Su lucha por la sobrevivencia y la libertad, IWGIA, Lima.

Labaka, Alejandro. 2003, Crónica Huaorani, CICAME, Quito.

Llorens, José A. 2002, "Etnicidad y censos: los conceptos básicos y sus aplicaciones", en: Bulletin de l'Institut Français d'Etudes Andines, 31(3), pp. 655-680.

Lucas, Kintto. 2000, "El regreso de los guerreros amazónicos", en http://www.ipsnoticias.net/2000/12/ecuadorel-regreso-de-los-guerreros-amazonicos/) (consultado el 6 de mayo de 2016).

Narváez, Iván. 1996, Huaorani Vs Maxus, Cecs, Quito.

Ministerio del Medio Ambiente. 1999, "Zonas Intangibles de la Amazonía ecuatoriana. Por la diversidad cultural y biológica”, Ministerio del Medio Ambiente-Unión Europea, Quito.

Prensa libre. 2013, "Ministerio modificó mapa de indígenas no contactados", en: http://www. democraciaycooperacion.net/espacio-colaborativo/america-latina/adjunta-tus-documentos-para-el/article/ ministerio-de-justicia-modifico (consultado el 20 de febrero de 2016).

Proaño, José. 2010, Voces de la selva. Noticias recientes sobre los pueblos indígenas aislados en la Amazonía ecuatoriana, Fundación Alejandro Labaka y otros, Quito.

2008, Pueblos indigenas en aislamiento voluntario: Tagaeiri-Taromenane en la Amazonía ecuatoriana, Abya Yala y Fundación Pachamama, Quito. 
Proaño García, José y Colleoni, Paola. 2010, Caminantes de la selva. Los pueblos en aislamiento de la Amazonía ecuatoriana, IWGIA, Quito.

Rogalski, Filip. 2003, Informe sobre la presencia de grupos indígenas en situación de aislamiento en la cuenca de los ríos: medio y alto Curaray, Arabela, Nashiño y Alto Napo. Iquitos.

Santos Ortiz de Villalba, Juan. 1996, Los últimos huaorani, CICAME, Quito.

Tagliani, Lino. 2004. También el sol muere. Cuatro años con los huaorani, CICAME, Quito.

Tassi, Giovanna (Coord.). 1992, Náufragos del Mar verde. La resistencia de los Huaorani a una integración impuesta, Abya-Yala, Quito.

Tessman, Günter, 1997, Die Indianer Nordost Perú. Quito, Abya-Yala.

Trujillo, Patricio. 2001, Salvajes, civilizados y civilizadores. La Amazonía ecuatoriana: el espacio de las ilusiones, Abya-Ayala, Quito.

TVECUADOR. 2013, "Los clanes Taromenane o Tagaeiri” [Reportaje televisivo].http://www.tvecuador.com/ index.php?option $=$ com_reportajes\&id $=242 \&$ view $=$ showcanal

Vera, Carlos. 2013, Secretos del Yasuni. Capítulo 1: la masacre de marzo [Documental].

2007, Taromenane, su historia y sus lanzas en manos de los Waorani. Cámara Oscura.

Villaverde, Xavier (Coord.). 2012, Los hijos del sol, FEPP, Quito. 